\title{
Complementary and Alternative Medicine for treating Low Back Pain with Teaching Exercise: A narrative review
}

\author{
Yeonhak Kim ${ }^{1,2}$, Gi-Young Yang ${ }^{3,4^{*}}$ \\ ${ }^{1}$ School of Korean Medicine, Pusan National University \\ ${ }^{2}$ Dept. of Korean Medicine Training, Changwon Jaseng Korean Medicine Hospital \\ ${ }^{3}$ Division of Clinical Medicine, School of Korean Medicine, Pusan National University \\ ${ }^{4}$ Department of Acupuncture \& Moxibustion Medicine, Pusan National University Korean Medicine Hospital
}

\begin{abstract}
Objectives: In modern society, many people have low back pain (LBP) and spinal diseases. About $80 \%$ of them experience severe LBP more than once in their lifetime. We can find studies on many Korean medicine-based treatments, such as acupuncture treatment for LBP and exercise therapy, which are effective in reducing the symptoms. This study focuses on the combined effect of both Korean medicine and exercise therapy for treating LBP. Method: For this review, we searched for articles focusing on pain and disability recovery in pre-clinical and clinical studies of extension and flexion exercise therapy related to LBP.

The search databases were as follows: PubMed, Google Scholar, and seven Korean electronic databases (Korea Citation Index (KCI), Korean studies Information Service System (KISS), Research Information Service System (RISS), Oriental Medicine Advanced Searching Integrated System (OASIS), DBPIA, National Digital Science Library (NDSL), and KOREAMED). The keywords were as follows: Korean Medicine, back pain, flexion exercise, extension exercise, McKenzie method, McKenzie exercise, Williams' flexion exercise, and Mechanical Diagnosis and Therapy. Results \& Conclusions: This review shows the usefulness of flexion and extension exercises for LBP treatment and effective patient education, but further studies are necessary.
\end{abstract}

$\overline{\text { Key Words }}$ : Low back pain, McKenzie method, Williams flexion exercise, Acupuncture

\section{Introduction}

In modern society, several people have low back pain (LBP) and spinal diseases. About $80 \%$ of them experience severe LBP more than once in their life time; it is the second most common disease in adults under the age of 45 years, the first being a cold. ${ }^{1)}$

Low back pain can lead to secondary diseases by lowering the quality of life and limiting exercise and daily activities. ${ }^{2)}$ Various treatment modalities for lumbar and spinal diseases exist in both Western and Korean medicine, and a medical approach appropriate for each disease is required. ${ }^{3)}$

The diagnostic classification of low back pain is as follows: Specific spinal pathology, Nerve root pain/radicular pain, and Non-specific low back pain. ${ }^{4}$ Among these, in the case of non-specific low back pain, 'Red flags; There is

\footnotetext{
- Received : 15 October 2020

- Revised : 1 December 2020

- Accepted : 1 December 2020

- Correspondence to : Gi-Young Yang

Division of Clinical Medicine, School of Korean Medicine, Pusan National University

20 Geumo-ro, Mulgeum-eup, Yangsan, Gyeongnamdo 50612, Republic of Korea

Tel : +82-55-360-5963, E-mail :ygy@pnu.edu
} 
no fracture, tumour, infections, systemic inflammatory disease' and is not a Radicular Syndrome. $^{5)}$

In addition, low back pain is divided into Acute (for less than 4 weeks), Subacute (for 4-12 weeks), and Chronic (for more than 12 weeks), based on the onset and duration, according to the ACP Guideline. ${ }^{6}$ According to the NASS standards, low back pain is divided into Acute (for less than 6 weeks), Subacute for (6 to 12 weeks), and Chronic (for more than 12 weeks). ${ }^{7)}$

Various treatments are performed for patients with chronic low back pain (C-LBP). However, when previous treatments are not effective, patients eventually visit the Korean medical clinic. According to the National Statistical Office (NSO), among the reasons for outpatient treatment in Korean medical clinics in 2014, low back pain accounted for $17.77 \%$, which was the highest rate of use among single diseases, excluding other classifications. ${ }^{8)}$ Studies have shown that practitioners tend to choose therapies such as acupuncture, Korean medicine physical therapy, moxibustion, cupping, use of a pack of prepared herbal medicine, Chuna manual therapy, herbal decoction, and pharmacopuncture, in this order. ${ }^{9)}$

The effectiveness of acupuncture for LBP has already been proved, and even in the guidelines ${ }^{6}$, 7,10) for LBP published in Europe and the United States, acupuncture (acupuncture treatment, laser acupuncture) is a Grade- $\mathrm{A}$ recommendation for C-LBP.

Exercise therapy for LBP can be broadly divided into extension exercises and flexion exercises. The representative exercise for extension is the 'McKenzie Method', and the flexion counterpart is 'Williams Flexion exercise'. Our aim was to check the actual effectiveness of exercise therapy that can enhance the effectiveness of Korean medicine treatment for low back pain. In addition, a summary of the exercises, which are actually applicable to patients receiving Korean medicine treatment has been included.

\section{Methods}

For this review, we searched the existing literature focusing on clinical studies depicting the effect of exercise on LBP. The search keywords included Korean Medicine, LBP, Flexion exercise, Extension exercise, McKenzie method, Williams' flexion exercise, and Mechanical Diagnosis and Therapy, and the following databases were searched: PubMed, Google Scholar electronic databases, Korea Citation Index (KCI), Korean Studies Information Service System (KISS), Research Information Service System (RISS), Oriental Medicine Advanced Searching Integrated System (OASIS), DBPIA, National Digital Science Library (NDSL), and KOREAMED.

\section{Use of exercise for treating LBP}

The summary of the details of exercises used for treating LBP are shown in Table 1.

\section{Flexion exercise for LBP: Williams flexion exercises}

Williams' exercises (Williams' lumbar flexion exercises, lumbar flexion exercises) mainly use flexion for exercise therapy. This method was first proposed by Paul C. Williams in 1937. Williams thought that LBP is caused by the 
compression of the intervertebral discs due to incorrect posture, which is the result of a lack of flexion exercises in daily lives.

The Williams flexion exercise consists of 7 types of exercises (pelvic tilt exercises, partial sit-ups, single and bilateral knee-to-chest, hamstring stretching, standing lunges, seated trunk flexion, and full squats), through which it improves lumbar flexion and strengthens the gluteal and abdominal muscles to recover from LBP. ${ }^{11)}$ In fact, one study showed increased flexibility in the hamstrings, gluteal flexors, and lumbar extensors and decreased LBP. ${ }^{12)}$

\section{Extension exercise for LBP: McKenzie Method}

In exercise therapy, the representative method of extension exercise is the McKenzie exercise. The McKenzie exercise is a workout method proposed by Robin McKenzie, a physical therapist in New Zealand. It categorizes LBP into three mechanical syndromes (posture, function, and regression) and other diseases (spinal stenosis, hip pain, sacroiliac joint pain, mechanically inconclusive diseases, spondylolisthesis, and chronic pain), and proceeds with a suitable exercise method. ${ }^{13)}$ McKenzie believed that a slouching posture usually moves the disc nucleus backwards, causing LBP. He aimed to increase the strength of the extensors in the lower back. There is a theoretical basis for pain relief upon moving the intervertebral disc forward through the extension of the lower back. ${ }^{14)}$

McKenzie's exercise therapy is classified into three mechanical syndromes through mechanical loading strategies (MLS), which are evaluated using the Visual Analogue Scale (VAS) for pain at the base point, pain at the extension point, and pain after extension. McKenzie suggested 25 exercises to treat LBP, based on MLS. Most exercises focus on extension, but flexion exercises are also performed as needed. ${ }^{15)}$ Several studies have shown that McKenzie's exercise therapy improves LBP, especially Acute LBP. ${ }^{16,17)}$

\section{Summary of the effects of exercise treatment on LBP}

This review summarizes the effects of exercise, including flexion and extension exercises, in patients with low back pain (Table 1, Table 2). In addition to McKenzie's and Williams' exercise therapy, all similar exercises are classified according to pain, disability, and muscle strength. The actual methods used for the McKenzie and Williams exercise therapies in each study are summarized in Table 3 .

A summary of the studies on each exercise therapy showed that both the pain and disability levels decreased. It was more important to perform one of the exercises than to have the flexion and extension movements. Various exercises using both the flexors and extensors reduced pain and disability.

Some studies state that the McKenzie method is excellent $\left(\mathrm{Ahn}^{18)}\right.$, Qurat-ul-Ain et $\mathrm{al}^{30)}$, Petersen et $\mathrm{al}^{33)}$ ), while others state that Williams' flexion exercise is better (Jeganathan et $\mathrm{al}^{31)}$ ).

Pain and disability reduction by McKenzie's and Williams' exercise therapies could not be evaluated as superior to that by specific exercise therapy. However, Mackenzie's exercise may be a better choice because both extensors and flexors improve muscle strength.

Moreover, the results of comparing pain and disability reduction between a 'trained group on exercise therapy' and a 'control group' showed 
greater pain and disability reduction with training. ${ }^{36)}$

The summary of McKenzie and Williams' exercise therapy used in each paper was invalidated if there was no detail, and if the detailed description of the motion was in accordance with each exercise, a point was used to grade each movement. Mackenzie's exercise therapy included lying prone, lying prone in extensions, sustained extensions, posture correction, extension mobility, extension in a standing position, slouch over correct/correcting, and flexion. Williams' exercise therapy used pelvic tilt, single knee to chest, double knee to chest,

Table 1. Effects of Flexion and Extension Exercise on LBP patients : Focusing on Pain and Disability

\begin{tabular}{|c|c|c|c|c|c|c|c|}
\hline \multirow{2}{*}{$\begin{array}{l}\text { Article } \\
\text { No. }\end{array}$} & \multicolumn{2}{|c|}{ Exercise method } & \multicolumn{2}{|c|}{ Result } & \multirow[b]{2}{*}{ Evaluation } & \multirow{2}{*}{$\begin{array}{c}\text { Subjects of LBP } \\
\text { Patient }\end{array}$} & \multirow[b]{2}{*}{ Author } \\
\hline & $\begin{array}{l}\text { Flexion } \\
\text { Exercise }\end{array}$ & $\begin{array}{l}\text { Extension } \\
\text { Exercise }\end{array}$ & Pain & Disability & & & \\
\hline 1 & o & o & improvement & improvement & Medx, OLBPDQ & C-LBP & $\mathrm{Ahn}^{18}$ \\
\hline 2 & $\mathrm{x}$ & o & improvement & $(-)$ & OLBPDQ & $\begin{array}{l}\text { HIVD, Lumbar } \\
\text { sprain and strain }\end{array}$ & Kim Ji-Sun etc ${ }^{19}$ \\
\hline 3 & $\mathrm{x}$ & $\mathrm{x}$ & improvement & improvement & VAS, OLBPDQ & C-LBP & Koo Hee-Seo ${ }^{20}$ \\
\hline 4 & $\mathrm{o}$ & o & improvement & improvement & $\begin{array}{c}\text { ODI, NRS, } \\
\text { OLBPDQ, FABQ }\end{array}$ & $\begin{array}{c}\text { A-LBP, Sub } \\
\text { A-LBP }\end{array}$ & Jeon $^{21}$ \\
\hline 5 & 0 & 0 & improvement & $(-)$ & PM & Nonspecific LBP & $\mathrm{Lee}^{22}$ \\
\hline 6 & o & $\mathrm{x}$ & improvement & improvement & VAS, RMDQ & C-LBP & Cambron $\mathrm{JA}^{23}$ \\
\hline 7 & 0 & 0 & improvement & improvement & PM & Nonspecific LBP & $\mathrm{Lee}^{24}$ \\
\hline 8 & 0 & 0 & improvement & improvement & VAS, ODI & C-LBP & $\mathrm{Choi}^{25}$ \\
\hline 9 & 0 & 0 & improvement & improvement & EMG & C-LBP & $\mathrm{Choi}^{26}$ \\
\hline 10 & 0 & 0 & improvement & $(-)$ & QLBPDQ & Nonspecific LBP & $\operatorname{Hur}^{27}$ \\
\hline 11 & $\mathrm{x}$ & 0 & improvement & improvement & VAS, K-ODI & C-LBP & $\mathrm{Choi}^{28}$ \\
\hline 12 & O & 0 & improvement & improvement & VAS, ODI & C-LBP & Kwon ${ }^{29}$ \\
\hline 13 & O & 0 & improvement & improvement & VAS, ODI & Nonspecific LBP & Qurat-ul-Ain ${ }^{30}$ \\
\hline 14 & 0 & 0 & improvement & $(-)$ & VAS & Mechanical LBP & Jeganathan, $A^{31}$ \\
\hline 15 & o & 0 & improvement & improvement & $\begin{array}{l}\text { VAS, sitting time, } \\
\text { flexion/lateral flexion, } \\
\text { lasseque test }\end{array}$ & Nonspecific LBP & Moldovan, Mircea ${ }^{32}$ \\
\hline 16 & o & o & improvement & $(-)$ & PM & $\begin{array}{l}\text { Sub A-LBP, } \\
\text { C-LBP }\end{array}$ & Petersen $T^{33}$ \\
\hline 17 & $\mathrm{x}$ & 0 & improvement & improvement & VAS, RMDQ & C-LBP & Johnson $\mathrm{OE}^{34}$ \\
\hline
\end{tabular}

* PM; Personal Method

Table 2. Effects of Flexion and Extension Exercise on LBP patients : Focusing on Extensors and Flexors improvement

\begin{tabular}{|c|c|c|c|c|c|}
\hline \multirow{2}{*}{ No. } & \multicolumn{2}{|c|}{ Muscle } & \multirow{2}{*}{ Subjects } & \multirow{2}{*}{ Evaluation } & \multirow{2}{*}{ Author } \\
\hline & Extensors & Flexors & & & \\
\hline 1 & improvement & $(-)$ & C-LBP & Medx muscle measurement & $\mathrm{Ahn}^{18}$ \\
\hline 2 & improvement & $(-)$ & C-LBP & Medx muscle measurement & $\mathrm{Choi}^{35}$ \\
\hline 3 & improvement & improvement & Nonspecific LBP & muscle measurement & $\mathrm{Lee}^{20}$ \\
\hline 4 & improvement & improvement & C-LBP & muscle measurement & $\mathrm{Choi}^{25}$ \\
\hline 5 & improvement & improvement & C-LBP & EMG & $\mathrm{Choi}^{26}$ \\
\hline 6 & improvement & improvement & C-LBP & muscle measurement & Kwon $^{29}$ \\
\hline
\end{tabular}


partial sit-up, hamstring stretch, hip flexor stretch, and squat. Exercise methods used at least five times were lying prone, lying prone in extension, sustained extensions, extensions in a standing position, flexion in lying (FIL), flexion in sitting, and pelvic tilt in Williams' exercise treatment (Table 3).

An effective method for treating diseases through a combination of exercise therapy and acupuncture has existed in Korean medicine as Dong-gi acupuncture (DGA), a kind of Dong-si acupuncture. The validity of using DGA for LBP by blood stasis and sprain has also been revealed. In particular, active DGA was more effective than passive DGA or common treatment. $^{37)}$ Another study compared simple acupuncture groups with acupuncture combined with DGA groups for treating cervical spondylosis. The difference in pain relief was not significant with acupuncture, but the time for pain relief was halved when compared with DGA (8.15 \pm 2.32 days) or simple acupuncture groups (13.25 \pm 4.62 days). ${ }^{38)}$ There was also a study on DGA, which combined flexion and extension exercises for lower lumbar pain. This study combined acupuncture treatment and exercise therapy based on the movement system impairment syndrome (MSIS), and organized the acupuncture points that may affect exercise therapy. ${ }^{39)}$

LBP is a common condition that affects about $80 \%$ of the population at least once in their lifetimes, and Korean medicine treatment, which accounts for about $18 \%$ of the outpatient treatment in Korean medicine clinics, is the main treatment. ${ }^{1)}$ Acupuncture is effective for treatment, and this has been demonstrated in guidelines for treatment in the United States and
Europe. $^{6,7)}$ Acupuncture-related treatment was recommended as Grade A, and the level of evidence was also observed as Grade Fair. Additionally, acupuncture treatment for C-LBP was evaluated as 'moderate level' for the Magnitude of Effect, which is well recognized to signify the level of pain relief; NSAIDs are 'Small to moderate level' and Tramadol is 'Moderate level'. The Non-Specific LBP guidelines are special in that they are also organized cases of better effects when combined with various non-invasive treatments.

By summarizing various studies, we have confirmed that the 'lying prone, lying prone in extension, sustained extension, extension in standing, flexion in lying (FIL), and flexion in sitting' of McKenzie's exercise therapy, and the 'pelvic tilt' of Williams' exercise reduces pain and disability (Table 1, Table 2, Table 3). It has already been confirmed that DGA, a Korean medicine treatment method, combines exercise therapy and acupuncture treatment.

Combining the information mentioned above, we can select an exercise motion within McKenzie's and Williams' exercises that is effective instead of utilizing other motions. Acupuncture and exercise therapy can simultaneously be performed at the acupuncture points where exercise is required. With further research, we can expect positive effects of combined acupuncture and exercise therapy rather than a common treatment and a reduction of treatment time for the same effect.

\section{Conflicts of Interest}

The authors have no conflicts of interest to declare. 
Table 3. McKenzie exercise and William's flexion exercise actually used in each research paper

\begin{tabular}{|c|c|c|c|c|c|c|c|c|c|c|c|c|c|c|c|c|c|c|c|}
\hline & \multirow{2}{*}{ Exercise Program } & \multirow{2}{*}{ SUM } & \multicolumn{17}{|c|}{ Article No. of Table. 1} \\
\hline & & & 1 & 2 & 3 & 4 & 5 & 6 & 7 & 8 & 9 & 10 & 11 & 12 & 13 & 14 & 15 & 16 & 17 \\
\hline \multirow{26}{*}{$\begin{array}{l}\text { McKenzie } \\
\text { exercise }\end{array}$} & Lying Prone & 6 & $\mathrm{x}$ & $\mathrm{o}$ & $\mathrm{x}$ & $\mathrm{x}$ & o & $\mathrm{x}$ & o & $\mathrm{x}$ & o & $\mathrm{x}$ & $\mathrm{o}$ & $\mathrm{x}$ & $\mathrm{x}$ & $\mathrm{x}$ & $\mathrm{x}$ & $\mathrm{x}$ & $\mathrm{o}$ \\
\hline & Lying Prone in Extension & 7 & o & o & $\mathrm{x}$ & $\mathrm{x}$ & o & $\mathrm{x}$ & o & $\mathrm{x}$ & o & $\mathrm{x}$ & o & $\mathrm{x}$ & $\mathrm{x}$ & $\mathrm{x}$ & $\mathrm{x}$ & $\mathrm{x}$ & o \\
\hline & Sustained extension & 8 & o & $\mathrm{o}$ & $\mathrm{x}$ & $\mathrm{x}$ & o & $\mathrm{x}$ & o & $\mathrm{x}$ & $\mathrm{o}$ & o & 0 & $\mathrm{x}$ & $\mathrm{x}$ & $\mathrm{x}$ & $\mathrm{x}$ & $\mathrm{x}$ & o \\
\hline & Posture Correction & 1 & $\mathrm{x}$ & $\mathrm{x}$ & o & $\mathrm{x}$ & $\mathrm{x}$ & $\mathrm{x}$ & $\mathrm{x}$ & $\mathrm{x}$ & $\mathrm{x}$ & $\mathrm{x}$ & $\mathrm{x}$ & $\mathrm{x}$ & $\mathrm{x}$ & $\mathrm{x}$ & $\mathrm{x}$ & $\mathrm{x}$ & $\mathrm{x}$ \\
\hline & $\begin{array}{l}\text { Extension in lying(with patient } \\
\text { overpressure) }\end{array}$ & 0 & $\mathrm{x}$ & $\mathrm{x}$ & $\mathrm{x}$ & $\mathrm{x}$ & $\mathrm{x}$ & $\mathrm{x}$ & $\mathrm{x}$ & $\mathrm{x}$ & $\mathrm{x}$ & $\mathrm{x}$ & $\mathrm{x}$ & $\mathrm{x}$ & $\mathrm{x}$ & $\mathrm{x}$ & $\mathrm{x}$ & $\mathrm{x}$ & $\mathrm{x}$ \\
\hline & $\begin{array}{l}\text { Extension in lying with Clinician } \\
\text { overpressure }\end{array}$ & 0 & $\mathrm{x}$ & $\mathrm{x}$ & $\mathrm{x}$ & $\mathrm{x}$ & $\mathrm{x}$ & $\mathrm{x}$ & $\mathrm{x}$ & $\mathrm{x}$ & $\mathrm{x}$ & $\mathrm{x}$ & $\mathrm{x}$ & $\mathrm{x}$ & $\mathrm{x}$ & $\mathrm{x}$ & $\mathrm{x}$ & $\mathrm{x}$ & $\mathrm{x}$ \\
\hline & $\begin{array}{l}\text { Extension in lying with belt } \\
\text { fixation }\end{array}$ & 0 & $\mathrm{x}$ & $\mathrm{x}$ & $\mathrm{x}$ & $\mathrm{x}$ & $\mathrm{x}$ & $\mathrm{x}$ & $\mathrm{x}$ & $\mathrm{x}$ & $\mathrm{x}$ & $\mathrm{x}$ & $\mathrm{x}$ & $\mathrm{x}$ & $\mathrm{x}$ & $\mathrm{x}$ & $\mathrm{x}$ & $\mathrm{x}$ & $\mathrm{x}$ \\
\hline & Extension mobilization & 1 & $x$ & $\mathrm{x}$ & o & $x$ & $\mathrm{x}$ & $\mathrm{x}$ & $\mathrm{x}$ & $\mathrm{x}$ & $x$ & $\mathrm{x}$ & $x$ & $x$ & $\mathrm{x}$ & $\mathrm{x}$ & $\mathrm{x}$ & $\mathrm{x}$ & $\mathrm{x}$ \\
\hline & Extension manipulation & 1 & $\mathrm{x}$ & $\mathrm{x}$ & $\mathrm{o}$ & $\mathrm{x}$ & $\mathrm{x}$ & $\mathrm{x}$ & $\mathrm{x}$ & $\mathrm{x}$ & $\mathrm{x}$ & $\mathrm{x}$ & $\mathrm{x}$ & $\mathrm{x}$ & $\mathrm{x}$ & $\mathrm{x}$ & $\mathrm{x}$ & $\mathrm{x}$ & $\mathrm{x}$ \\
\hline & Extension in standing & 5 & $\mathrm{x}$ & o & $\mathrm{x}$ & $\mathrm{x}$ & o & $\mathrm{x}$ & o & $\mathrm{x}$ & o & $\mathrm{x}$ & o & $\mathrm{x}$ & $\mathrm{x}$ & $\mathrm{x}$ & $\mathrm{x}$ & $\mathrm{x}$ & $\mathrm{x}$ \\
\hline & $\begin{array}{l}\text { Slouch over correct/correct } \\
\text { sitting posture }\end{array}$ & 1 & $\mathrm{x}$ & $\mathrm{x}$ & o & $\mathrm{x}$ & $\mathrm{x}$ & $\mathrm{x}$ & $\mathrm{x}$ & $\mathrm{x}$ & $\mathrm{x}$ & $\mathrm{x}$ & $\mathrm{x}$ & $\mathrm{x}$ & $\mathrm{x}$ & $\mathrm{x}$ & $\mathrm{x}$ & $\mathrm{x}$ & $\mathrm{x}$ \\
\hline & $\begin{array}{l}\text { Extension in lying with hips off } \\
\text { center }\end{array}$ & 0 & $\mathrm{x}$ & $\mathrm{x}$ & $\mathrm{x}$ & $\mathrm{x}$ & $\mathrm{x}$ & $\mathrm{x}$ & $\mathrm{x}$ & $\mathrm{x}$ & $\mathrm{x}$ & $\mathrm{x}$ & $\mathrm{x}$ & $\mathrm{x}$ & $\mathrm{x}$ & $\mathrm{x}$ & $\mathrm{x}$ & $\mathrm{x}$ & $\mathrm{x}$ \\
\hline & $\begin{array}{l}\text { Extension in lying with hips off } \\
\text { center with clinician } \\
\text { overpressure(sagittal or lateral) }\end{array}$ & 0 & $\mathrm{x}$ & $\mathrm{x}$ & $\mathrm{x}$ & $\mathrm{x}$ & $\mathrm{x}$ & $\mathrm{x}$ & $\mathrm{x}$ & $\mathrm{x}$ & $\mathrm{x}$ & $\mathrm{x}$ & $\mathrm{x}$ & $\mathrm{x}$ & $\mathrm{x}$ & $\mathrm{x}$ & $\mathrm{x}$ & $\mathrm{x}$ & $\mathrm{x}$ \\
\hline & $\begin{array}{l}\text { Extension mobilization with hips } \\
\text { off center }\end{array}$ & 0 & $\mathrm{x}$ & $\mathrm{x}$ & $\mathrm{x}$ & $\mathrm{x}$ & $\mathrm{x}$ & $\mathrm{x}$ & $\mathrm{x}$ & $\mathrm{x}$ & $\mathrm{x}$ & $\mathrm{x}$ & $\mathrm{x}$ & $\mathrm{x}$ & $\mathrm{x}$ & $\mathrm{x}$ & $\mathrm{x}$ & $\mathrm{x}$ & $\mathrm{x}$ \\
\hline & $\begin{array}{l}\text { Rotation mobilization in } \\
\text { extension }\end{array}$ & 0 & $\mathrm{x}$ & $\mathrm{x}$ & $\mathrm{x}$ & $\mathrm{x}$ & $\mathrm{x}$ & $\mathrm{x}$ & $\mathrm{x}$ & $\mathrm{x}$ & $\mathrm{x}$ & $\mathrm{x}$ & $\mathrm{x}$ & $\mathrm{x}$ & $\mathrm{x}$ & $\mathrm{x}$ & $\mathrm{x}$ & $\mathrm{x}$ & $\mathrm{x}$ \\
\hline & $\begin{array}{l}\text { Rotation manipulation in } \\
\text { extension }\end{array}$ & 0 & $\mathrm{x}$ & $\mathrm{x}$ & $\mathrm{x}$ & $\mathrm{x}$ & $\mathrm{x}$ & $\mathrm{x}$ & $\mathrm{x}$ & $\mathrm{x}$ & $\mathrm{x}$ & $\mathrm{x}$ & $\mathrm{x}$ & $\mathrm{x}$ & $\mathrm{x}$ & $\mathrm{x}$ & $\mathrm{x}$ & $\mathrm{x}$ & $\mathrm{x}$ \\
\hline & $\begin{array}{l}\text { Self-correction of lateral shift or } \\
\text { sidegliding }\end{array}$ & 0 & $\mathrm{x}$ & $\mathrm{x}$ & $\mathrm{x}$ & $\mathrm{x}$ & $\mathrm{x}$ & $\mathrm{x}$ & $\mathrm{x}$ & $\mathrm{x}$ & $\mathrm{x}$ & $\mathrm{x}$ & $\mathrm{x}$ & $\mathrm{x}$ & $\mathrm{x}$ & $\mathrm{x}$ & $\mathrm{x}$ & $\mathrm{x}$ & $\mathrm{x}$ \\
\hline & Manual correction of lateral shift & 0 & $\mathrm{x}$ & $\mathrm{x}$ & $\mathrm{x}$ & $\mathrm{x}$ & $\mathrm{x}$ & $\mathrm{x}$ & $\mathrm{x}$ & $\mathrm{x}$ & $\mathrm{x}$ & $\mathrm{x}$ & $\mathrm{x}$ & $\mathrm{x}$ & $\mathrm{x}$ & $\mathrm{x}$ & $\mathrm{x}$ & $\mathrm{x}$ & $\mathrm{x}$ \\
\hline & Flexion in lying(FIL) & 5 & $\mathrm{x}$ & $\mathrm{o}$ & $\mathrm{x}$ & $\mathrm{x}$ & o & $\mathrm{x}$ & 0 & $\mathrm{x}$ & o & $\mathrm{x}$ & 0 & $\mathrm{x}$ & $\mathrm{x}$ & $\mathrm{x}$ & $\mathrm{x}$ & $\mathrm{x}$ & $\mathrm{x}$ \\
\hline & Flexion in sitting & 5 & $\mathrm{x}$ & o & $\mathrm{x}$ & $\mathrm{x}$ & o & $\mathrm{x}$ & o & $\mathrm{x}$ & o & $\mathrm{x}$ & $\mathrm{o}$ & $\mathrm{x}$ & $\mathrm{x}$ & $\mathrm{x}$ & $\mathrm{x}$ & $\mathrm{x}$ & $\mathrm{x}$ \\
\hline & Flexion in standing(FIS) & 0 & $x$ & $\mathrm{x}$ & $\mathrm{x}$ & $\mathrm{x}$ & $\mathrm{x}$ & $x$ & $\mathrm{x}$ & $\mathrm{x}$ & $\mathrm{x}$ & $x$ & $\mathrm{x}$ & $\mathrm{x}$ & $\mathrm{x}$ & $\mathrm{x}$ & $x$ & $\mathrm{x}$ & $\mathrm{x}$ \\
\hline & $\begin{array}{l}\text { Flexion in lying with clinician } \\
\text { overpressure }\end{array}$ & 0 & $\mathrm{x}$ & $\mathrm{x}$ & $\mathrm{x}$ & $\mathrm{x}$ & $\mathrm{x}$ & $\mathrm{x}$ & $\mathrm{x}$ & $\mathrm{x}$ & $\mathrm{x}$ & $\mathrm{x}$ & $\mathrm{x}$ & $\mathrm{x}$ & $\mathrm{x}$ & $\mathrm{x}$ & $\mathrm{x}$ & $\mathrm{x}$ & $\mathrm{x}$ \\
\hline & Flexion in step standing(FISS) & 0 & $\mathrm{x}$ & $\mathrm{x}$ & $\mathrm{x}$ & $\mathrm{x}$ & $\mathrm{x}$ & $\mathrm{x}$ & $\mathrm{x}$ & $\mathrm{x}$ & $\mathrm{x}$ & $\mathrm{x}$ & $\mathrm{x}$ & $\mathrm{x}$ & $\mathrm{x}$ & $\mathrm{x}$ & $\mathrm{x}$ & $\mathrm{x}$ & $\mathrm{x}$ \\
\hline & Rotation in flexion & 0 & $\mathrm{x}$ & $\mathrm{x}$ & $\mathrm{x}$ & $\mathrm{x}$ & $\mathrm{x}$ & $\mathrm{x}$ & $\mathrm{x}$ & $\mathrm{x}$ & $\mathrm{x}$ & $\mathrm{x}$ & $\mathrm{x}$ & $\mathrm{x}$ & $\mathrm{x}$ & $\mathrm{x}$ & $\mathrm{x}$ & $\mathrm{x}$ & $\mathrm{x}$ \\
\hline & Rotation mobilization in flexion & 0 & $\mathrm{x}$ & $\mathrm{x}$ & $\mathrm{x}$ & $\mathrm{x}$ & $\mathrm{x}$ & $\mathrm{x}$ & $\mathrm{x}$ & $\mathrm{x}$ & $\mathrm{x}$ & $\mathrm{x}$ & $\mathrm{x}$ & $\mathrm{x}$ & $\mathrm{x}$ & $\mathrm{x}$ & $\mathrm{x}$ & $\mathrm{x}$ & $\mathrm{x}$ \\
\hline & Rotation manipulation in flexion & 0 & $\mathrm{x}$ & $\mathrm{x}$ & $\mathrm{x}$ & $\mathrm{x}$ & $\mathrm{x}$ & $\mathrm{x}$ & $\mathrm{x}$ & $\mathrm{x}$ & $\mathrm{x}$ & $\mathrm{x}$ & $\mathrm{x}$ & $\mathrm{x}$ & $\mathrm{x}$ & $\mathrm{x}$ & $\mathrm{x}$ & $\mathrm{x}$ & $\mathrm{x}$ \\
\hline \multirow{7}{*}{$\begin{array}{l}\text { William's } \\
\text { flexion } \\
\text { exercise }\end{array}$} & Pelvic tilt & 5 & o & $\mathrm{x}$ & o & $\mathrm{x}$ & o & $\mathrm{x}$ & o & $\mathrm{x}$ & o & $\mathrm{x}$ & $\mathrm{x}$ & $\mathrm{x}$ & $\mathrm{x}$ & $\mathrm{x}$ & $\mathrm{x}$ & $\mathrm{x}$ & $\mathrm{x}$ \\
\hline & Single knee to chest & 2 & o & $\mathrm{x}$ & $\mathrm{o}$ & $\mathrm{x}$ & $\mathrm{x}$ & $\mathrm{x}$ & $\mathrm{x}$ & $\mathrm{x}$ & $\mathrm{x}$ & $\mathrm{x}$ & $\mathrm{x}$ & $\mathrm{x}$ & $\mathrm{x}$ & $\mathrm{x}$ & $\mathrm{x}$ & $\mathrm{x}$ & $\mathrm{x}$ \\
\hline & Double knee to chest & 3 & $\mathrm{o}$ & $\mathrm{x}$ & $\mathrm{x}$ & $\mathrm{x}$ & $\mathrm{x}$ & $\mathrm{x}$ & 0 & $\mathrm{x}$ & o & $\mathrm{x}$ & $\mathrm{x}$ & $\mathrm{x}$ & $\mathrm{x}$ & $\mathrm{x}$ & $\mathrm{x}$ & $\mathrm{x}$ & $\mathrm{x}$ \\
\hline & Partial sit-up & 4 & 0 & $\mathrm{x}$ & $\mathrm{x}$ & $\mathrm{x}$ & o & $\mathrm{x}$ & 0 & $\mathrm{x}$ & o & $\mathrm{x}$ & $\mathrm{x}$ & $\mathrm{x}$ & $\mathrm{x}$ & $\mathrm{x}$ & $\mathrm{x}$ & $\mathrm{x}$ & $\mathrm{x}$ \\
\hline & Hamstring stretch & 3 & $\mathrm{x}$ & $\mathrm{x}$ & $\mathrm{x}$ & $\mathrm{x}$ & o & $\mathrm{x}$ & o & $\mathrm{x}$ & o & $\mathrm{x}$ & $\mathrm{x}$ & $\mathrm{x}$ & $\mathrm{x}$ & $\mathrm{x}$ & $\mathrm{x}$ & $\mathrm{x}$ & $\mathrm{x}$ \\
\hline & Hip Flexor stretch & 3 & $\mathrm{x}$ & $\mathrm{x}$ & $\mathrm{x}$ & $\mathrm{x}$ & o & $\mathrm{x}$ & 0 & $\mathrm{x}$ & o & $\mathrm{x}$ & $\mathrm{x}$ & $\mathrm{x}$ & $x$ & $\mathrm{x}$ & $\mathrm{x}$ & $\mathrm{x}$ & $\mathrm{x}$ \\
\hline & Squat & 3 & 0 & $\mathrm{x}$ & $\mathrm{x}$ & $\mathrm{x}$ & $\mathrm{x}$ & $\mathrm{x}$ & o & $\mathrm{x}$ & o & $\mathrm{x}$ & $\mathrm{x}$ & $\mathrm{x}$ & $\mathrm{x}$ & $\mathrm{x}$ & $\mathrm{x}$ & $\mathrm{x}$ & $\mathrm{x}$ \\
\hline
\end{tabular}




\section{Acknowledgements}

This work was supported by clinical research grant from Pusan National University Hospital in 2020.

We would like to thank Editage (www.editage.co.kr) for English language editing.

\section{References}

1. Walker BF. The prevalence of low back pain: a systematic review of the literature from 1966 to 1998, J Spinal Disord. 2000; 13:205-17.

2. Otávio Turolo da SilvaAndrei Fernandes JoaquimAlpesh A. Patel, Fundamentals of Neurosurgery:Springer, Cham. 2019;191-199.

3. Koh W, Kang K, Lee YJ, Kim M-r, Shin JS, Lee J, et al. Impact of acupuncture treatment on the lumbar surgery rate for low back pain in Korea: A nationwide matched retrospective cohort study, . PLoS ONE. 2018; 13(6): e0199042.

4. Waddell G. A new clinical model for the treatment of low-back pain. Spine. 1987; 12(7): 632-44.

5. ROBERT L. BRATTON, Assessment and Management of Acute Low Back Pain.Am Fam Physician.1999;60(8):2299-2306.

6. Qaseem A, Wilt TJ, McLean RM, Forciea MA. Clinical Guidelines Committee of the American College of Physicians. Noninvasive Treatments for Acute, Subacute, and Chronic Low Back Pain: A Clinical Practice Guideline From the American College of Physicians. Ann Intern Med. 2017;166(7):514-530.

7. D. Scott Kreiner et al. Evidence-Based Clinical Guidelines for Multidisciplinary
Spine Care: Diagnosis \& Treatment of Low Back Pain, North American Spine Society, 2020:1-217.

8. Survey on the use of Korean medicine and consumption of oriental medicine: Reasons for using Korean medicine outpatient treatment, Statistical Office, 2014; http://kosis.kr/statHtml/ statHtml.do?orgId=117\&tblId=DT_11787N_ 0012\&conn_path $=$ I2

9. Survey on the use of oriental medicine and consumption of oriental medicine: treatment methods by symptom, Statistical Office, 2014; http://kosis.kr/statHtml/statHtml.do?orgId $=117 \&$ tblId=DT_11787N_0013\&conn_path=I2

10. O. Airaksinen, J. I. Brox, C. Cedraschi, J. Hildebrandt, J. Klaber-Moffett, F. Kovacs, A. F. Mannion, S. Reis, J. B. Staal, H. Ursin, G. Zanoli, and On behalf of the COST B13 Working Group on Guidelines for Chronic Low Back Pain. European guidelines for the management of chronic nonspecific low back pain.; Eur Spine J. 2006:S192-S300.

11. Dydyk AM, Sapra A. Williams Back Exercises. StatPearls [Internet]. 2020 Jul 10. PMID: 31855385.

12. Fatemi R, Javid M, Najafabadi EM. Effects of William training on lumbosacral muscles function, lumbar curve and pain. Journal of Back and Musculoskeletal Rehabilitation. $2015 ; 28(3): 591-597$.

13. McKenzie R, Stephen May, The Lumbar Spine Mechanical Diagnosis and Therapy, Vol. 1\&2. : Spinal publications. 2003:1-728.

14. Han-Ki Lee, PT, PhD, Young-Hyun Cho, PT, MS, Jun-Cheol Lee. The Effect of Improve the Waist Flexibility, the Waist Muscular Strength and the Waist Balance which Grafted in William \& McKenzie 
Exercise with Swiss Ball, J Korean Soc Phys Med. 2013;8(4):479-487

15. Manual Therapy Institute, Advanced Lumbar Spine; Manual Therapy Institute Publications: 3-38.

16. Lam OT, Strenger DM, Chan-Fee M, Pham PT, Preuss RA, Robbins SM. Effectiveness of the McKenzie Method of Mechanical Diagnosis and Therapy for Treating Low Back Pain: Literature Review With Meta-analysis, J Orthop Sports Phys Ther. 2018;48(6):476-490.

17. Long, Audrey \& Donelson, Ronald \& Fung, Tak. Does it Matter Which Exercise? A Randomised Control Trial of Exercise for Low Back Pain, Spine. 2004;29(23):2593 -2602 .

18. Wang Hoon Ahn. (The)effect of lumbar flexion exercise on the extensor muscle strength and pain in idiopathic low back pain patients: Thesis (Master's) Korea University. 2005:27-31.

19. Kim, Ji-Sun \& Kim, Myung-Hoe \& Yu, Byoung-Kyu. The Effect of Extension Exercise on the Reduction of Pain in Patients with Low Back Pain, Korean Physical Therapy Science. 1996;3(1):843-453.

20. Koo, Hee-Seo. The Effects of Australian Stabilization Exercises for Decreasing Dysfunction and Pain in Chronic Back Pain Patients, Korean Physical Therapy Science. 2009;16(4):39-47.

21. Jeon, Ji-hye \& Kim, Suhn-yeop. Comparison of Lumbar Stabilization Exercises and Gluteal Strengthening Exercises on Pain, Disability and Psychosocial Factors in Low Back Pain Patients with Lumbar Instability, Korean Academy of Orthopedic Manipulative Physical
Therapy. 2017;23(2):33-44.

22. Lee, Han-Ki \& Cho, Young-Hyun \& Lee, Jun-Cheol. The Effect of Improve the Waist Flexibility, the Waist Muscular Strength and the Waist Balance which Grafted in William \& McKenzie Exercise with Swiss Ball, Journal of the Korean Society of Physical Medicine. 2013;8(4):479-487.

23. Cambron JA, Gudavalli MR, Hedeker D, McGregor M, Jedlicka J, Keenum M, Ghanayem AJ, Patwardhan AG, Furner SE. One-year follow-up of a randomized clinical trial comparing flexion distraction with an exercise program for chronic low-back pain, J Altern Complement Med. 2006;12(7): 659-68.

24. AhReum Lee, HanSuk Jung, BoRam Eo. The effects of overweight and low back pain patient by McKenzie and William exercise, Research Journal of Complementary and Alternative Medicine. 2016;7(7):79-89.

25. Choi, Soon Young. The Effect of Exercise Program on Chronic Low Back Pain in Female Teachers of Elementary School, Korean J Women Health Nurs. 2001;7(2): 169-187.

26. Choi Young-Deog, Lee kwang-Sik,Yoon Cheul-soo. The effect of the McKenzie exercise and the Williams exercise on the reduction of low back pain and on the changes of EMG, The journal of Korean academy of physical therapist. 2000;7(2); 663-682.

27. Hur, Jin-gang. Effect on Thoracic Exercise Programs in Employees With Chronic Low Back Pain, Korean Research Society of Physical Therapy. 2005;12(2): 44-57.

28. Choi, Jong-Won \& Kim, Min-Young \& 
Kim, Sung-Hwa \& Son, Bo-Hyun \& Lee, Su-Min \& Lee, Yu-Jeong \& Jang, Da-Vin \& Je, Hyo-Min \& Kim, Ki-Hyun. Effects of a Horse Riding Simulator, Gym-ball and McKenzie Exercises on Back Pain and Balance in Patients with Chronic Back Pain in Their 20s. Journal of The Korean Society of Physical Medicine. 2019;14(3):117-126 117-126.

29. Kwon Hwi-ryeon.The effects of lumbar self-exercise program on physical strength factors(strength, flexibility), pain and OSI in patients with the chronic low back pain: Thesis (Master's) Ewha University. 2005:1-61.

30. Qurat-ul-Ain, and Iqra ishaq. COMPARISON BETWEEN THE EFFECTIVENESS OF MCKENZIE EXTENSION EXERCISES AND WILLIAM FLEXION EXERCISES FOR TREATMENT OF NON-SPECIFIC LOW BACK PAIN. Journal of University Medical \& Dental College. 2017;8(3):68-72.

31. Jeganathan, A., Aishwarya Kanhere, and R. Monisha. A comparative study to determine the effectiveness of the mckenzie exercise and williams exercise in mechanical low back pain. Research Journal of Pharmacy and Technology 2018;11(6):2440-2443.

32. Moldovan, Mircea. Therapeutic Considerations and Recovery in Low Back Pain: Williams vs McKenzie. Timisoara Physical Education and Rehabilitation Journal. 2012;5(9): 2012: 58

33. Petersen T, Kryger P, Ekdahl C, Olsen S, Jacobsen S. The effect of McKenzie therapy as compared with that of intensive strengthening training for the treatment of patients with subacute or chronic low back pain: A randomized controlled trial. Spine
(Phila Pa 1976). 2002 Aug 15;27(16):1702-9.

34. Johnson OE, Adegoke BO, Ogunlade SO. Comparison of four physiotherapy regimens in the treatment of long-term mechanical low back pain. J Jpn Phys Ther Assoc. 2010;13(1):9-16.

35. Choi, Young-Deog. The Effect on Chronic Low Back Pain Patient Through Lumbar Extension Muscular Strength Training, Journal of Korean Physical Therapy Science. 1995;2(4):779-783.

36. Johnson, Olubusola E et al. Comparison of four physiotherapy regimens in the treatment of long-term mechanical low back pain. Journal of the Japanese Physical Therapy Association;2010;13(1): 9-16.

37. Soon Jung Kwon, Kee Hyun Kim, Kyung Nam Kim, Mi Suk Kang, Seong No Lee, Hyeon Seo Hwang, Ho Sueb Song, Hyung Ho Lim, Young Sang Song, Koang Hyun Ahn, Im Jung Gyun. Original Article : The Study of Effect and Safety related to Dong gi Acupuncture (DGA) and Complex therapy on Lumbago due to blood stasis and sprain. The Journal Of Korean Acupuncture \& Moxibustion Medicine Society, 2002;19(3); 107-114.

38. Ben-hua LUO, Jing-xian HAN, Cervical Spondylosis Treated by Acupuncture at Ligou (LR 5) Combined with Movement Therapy, Journal of Traditional Chinese Medicine; 2010;30(2) :113-117.

39. Youn Woo Seok, Park Young Jae, Park Young Bae. Dong-Qi Therapy of Dong-Si Acupuncture to Movement System Impairment Syndrome of Lumbar Spine and Knee, J Acupunct Res. 2013;30(1):13-22. 


\section{ORCID}

YEONHAK KIM https://orcid.org/0000-0003-2025-000X

Gi Young Yang https://orcid.org/0000-0002-9468-8904 